\title{
The effectiveness of a Housing First adaptation for ethnic minority groups: findings of a pragmatic randomized controlled trial
}

Vicky Stergiopoulos ${ }^{1,2,3}$, Agnes Gozdzik², Vachan Misir ${ }^{2}$, Anna Skosireva ${ }^{2}$, Aseefa Sarang ${ }^{4}$, Jo Connelly ${ }^{5}$, Adam Whisler ${ }^{1}$ and Kwame McKenzie ${ }^{1,3,6^{*}}$

\begin{abstract}
Background: Little is known about the effectiveness of Housing First (HF) among ethnic minority groups, despite its growing popularity for homeless adults experiencing mental illness. This randomized controlled trial tests the effectiveness of a HF program using rent supplements and intensive case management, enhanced by anti-racism and anti-oppression practices for homeless adults with mental illness from diverse ethnic minority backgrounds.

Methods: This unblinded pragmatic field trial was carried out in community settings in Toronto, Canada. Participants were 237 adults from ethnic minority groups experiencing mental illness and homelessness, who met study criteria for moderate needs for mental health services. Participants were randomized to either adapted HF ( $n=135)$ or usual care $(n=102)$ and followed every 3 months for 24 months. The primary study outcome was housing stability; secondary outcomes included physical and mental health, social functioning, quality of life, arrests and health service use. Intention to treat statistical analyses examined the effectiveness of the intervention compared to usual care.

Results: During the 24-month study period, HF participants were stably housed a significantly greater proportion of time compared to usual care participants, $75 \%$ (95\% Cl 70 to 81 ) vs. $41 \%$ (95 \% Cl 35 to 48), respectively, for a difference of $34 \%, 95 \% \mathrm{Cl} 25$ to 43 . HF also led to improvements in community integration over the course of the study: the change in the mean difference between treatment groups from baseline to 24-months was significantly greater among HF participants compared to those in usual care (change in mean difference $=2.2$, $95 \% \mathrm{Cl} 0.06$ to 4.3). Baseline diagnosis of psychosis was associated with reduced likelihood of being housed $\geq 50 \%$ of the study period ( $\mathrm{OR}=0.37,95 \% \mathrm{Cl} 0.18$ to 0.72 ).
\end{abstract}

Conclusion: Housing First enhanced with anti-racism and anti-oppression practices can improve housing stability and community functioning among ethnically diverse homeless adults with mental illness.

Trial registration: International Standard Randomized Control Trial Number Register Identifier: ISRCTN42520374, assigned August 18, 2009.

Keywords: Homelessness, Mental illness ethnic minority, Housing

\footnotetext{
*Correspondence: Kwame.McKenzie@camh.ca; Kwame_McKenzie@camh.ca

${ }^{1}$ Centre for Addiction and Mental Health, Toronto, ON, Canada

${ }^{3}$ Department of Psychiatry, University of Toronto, Toronto, ON, Canada

Full list of author information is available at the end of the article
} 


\section{Background}

Ethnic differences in access to, use and outcomes of mental health care have been reported in the US, UK and Canada [1-3]. The literature on health disparities faced by individuals from ethnic minority groups experiencing both homelessness and mental illness, or how to address them, however, is scant $[4,5]$. Homeless individuals experience high rates of mental illness, often in conjunction with substance misuse [6]; this population experiences poor access to care, increased morbidity and premature mortality [6], with ethnic disparities possibly contributing to additional disadvantage. Housing First (HF) and similar consumer-driven programs have emerged as effective interventions for homeless adults experiencing mental illness, offering participants immediate access to independent housing and mental health support services [7, 8], without the strict requirements of "housing readiness" (sobriety and acceptance of psychiatric treatment) imposed by more traditional "treatment first" interventions [9]. The At Home/Chez Soi study was a randomized field trial of HF conducted between 2009 and 2013 in five sites across Canada to assess the effectiveness of HF with either assertive community treatment or intensive case management for homeless adults with mental illness in diverse service delivery contexts [10]. In Toronto, Canada's largest and most ethnically diverse urban centre, in addition to testing the effectiveness of HF for a representative sample of homeless adults with mental illness, an additional trial examined the effectiveness of a HF adaptation among ethnic minority groups with moderate needs for mental health support services. In consultation with local community agencies, an adapted HF intervention was developed which incorporated anti-racist and anti-oppressive practices with the aim of improving outcomes for black and ethnic minority participants by recognizing and addressing the impact of racism and oppression on their lives [11]. This paper examines the effectiveness of the adapted HF intervention, compared to usual care, in keeping homeless people from black and minority ethnic groups in stable housing and in improving their social functioning, quality of life and physical and mental health.

\section{Methods}

\section{Study design and setting}

We conducted a two-year randomized controlled trial in Toronto, part of the At Home/Chez Soi cross-site project, in order to assess the effectiveness of HF with intensive case management for homeless adults with mental illness from diverse ethnic minority groups. The protocol for the overall At Home/Chez Soi project [10] and the Toronto site specifically [12] have been described previously. Prior to randomization, all participants were first stratified either to a high needs or moderate needs group based on their level of need for mental health services. At the Toronto site, participants in the moderate need group were further stratified by ethnicity (belonging to ethnic minority groups or not). This article focuses exclusively on study outcomes among the sample of moderate needs participants who belong to an ethnic minority group.

The study was approved by the Research Ethics Board (\#09-208) of St. Michael's Hospital in Toronto, and was registered with the International Standard Randomized Control Trial Number (ISRCTN 42520374). All study participants provided written informed consent.

\section{Participants}

All At Home/Chez Soi participants met three inclusion criteria: a) $\geq 18$ years old; b) absolutely homeless (having no fixed place to stay for at least the past seven nights with little likelihood of finding a place in the upcoming month) or precariously housed (currently occupying a single room in a multi-tenant building or house with shared common areas including bathroom and kitchen [13] or a hotel/motel as a primary residence, and having a history of two or more episodes of absolute homelessness in the past year); and c) presence of a current mental disorder with or without a co-existing substance use disorder, based on the DSM-IV [14] criteria in the Mini International Neuropsychiatric Interview 6.0 (MINI) [15]. Eligible diagnoses included: a) major depressive episode; b) manic or hypomanic episode; c) mood disorder with psychotic features; d) panic disorder; e) posttraumatic stress disorder; or f) psychotic disorder. Diagnosis of a substance use disorder alone did not qualify participants for study entry. Participants were excluded from the study if they: a) were relatively homeless (people who are residing in conditions that do not meet basic standards but who are not absolutely homeless or precariously housed, including those living in overcrowded or hazardous housing, transitional housing such as shelters for domestic abuse, long-term institutions, couch surfing, and people at risk of homelessness or lacking a dwelling for a short period of time due to disasters such as fire or economic situations) [10]; b) lacked a diagnosis of a serious mental disorder; c) had no legal status in Canada; or d) were current recipients of mental health supports via assertive community treatment $(\mathrm{ACT})$ or intensive case management (ICM) services [10].

Participant recruitment was based on referrals from both community social service agencies and acute care services. A targeted recruitment strategy was employed to ensure that the broader study sample adequately represented the target population [16], and all participants were assessed by the intake coordinator who determined 
study eligibility. Given this study's focus on black and ethnic minority participants, we recruited extensively among diverse ethnic minority groups through outreach to ethnoracial agencies serving this population.

\section{Stratification and randomization}

Prior to randomization, all eligible participants for the larger trial were stratified into either a high need or moderate need group, indicating their need for mental health services. The stratification algorithm defined high need participants as those who had: 1) community functioning scores from the Multnomah Community Ability Scale (MCAS) $\leq 62$ [17]; and 2) a diagnosis of current psychotic or bipolar disorder based on the MINI [15], in addition to meeting one of the following three criteria: i) $\geq 2$ hospitalizations for mental illness in any one year in the last 5 years; or ii) diagnosis of comorbid substance use based on the MINI; or iii) recent arrest(s) or incarceration(s) in the past 6 months. High needs participants were randomized to HF with ACT or a usual care group. All other participants were considered to have moderate need for mental health supports services.

Moderate needs participants were further stratified by ethnicity. Self-reported ethnicity was measured using a form validated locally [18], which asked people to select their ethnicity from one of 15 groups based on race and geographical origin: those who selected Black African (e.g. Ghana, Kenya, Somalia), Black Canadian/American, Black Caribbean (e.g. Jamaica, Trinidad, Tobago), East Asian (e.g. China, Japan, Korea), Indian-Caribbean (e.g. Guyana with origins in India), Latin American (e.g. Argentina, Chile, Costa Rica), Middle Eastern (e.g. Egypt, Iran, Israel, Palestine), South Asian (e.g. India, Pakistan, Sri Lanka) and South East Asian (e.g. Malaysia, Philippines, Vietnam) or who reported mixed background that included at least one of the ethnic groups listed above were considered eligible for the adapted HF program. Participants were excluded if they self-identified as Aboriginal, White (European or Canadian) or of a mixed ethnicity that did not include one of the specified groups listed above.

Moderate need participants not belonging to an ethnic minority group were allocated to a regular HF with ICM program or usual care control group. All moderate needs participants belonging to an ethnic minority group $(n=237)$ were randomized to the adapted HF intervention or usual care. A small group of participants $(n=33)$ randomized to the adapted HF program did not receive the adapted intervention either because there was no space available or they requested a non-ethno-racial focused program, and instead received services from the regular HF with ICM program.

Randomization took place via adaptive randomization procedures using a laptop computer connected to the study data management centre: by continuously adjusting the probability of allocation to each treatment group based on existing group assignment, this procedure can produce better balance between treatment groups than strict randomization in small and moderate sized studies $[10,19]$. Several aspects of the study prohibited blinding, including the nature of the administered questionnaires (detailed housing history and service use), location of participant interviews (some participants elected to be interviewed at their place of residence) and follow-up procedures (locating participants often required aid from case managers or community workers, where consent was given). As a result, masking follow-up data was also not possible. However, the allocation algorithm was concealed from both the participants and the research staff. Following randomization, participants allocated to the intervention group were immediately connected with their treatment teams, while usual care participants and their referral sources were provided with information about other existing services.

\section{Interventions}

The adapted HF intervention was developed uniquely for the Toronto site with ICM services provided by a mental health agency exclusively serving ethnic minority groups using anti-racist and anti-oppressive frameworks of practice. The model has been described in greater detail elsewhere [11]. The development of the adapted model was informed by practices within the leading agency and a review of the literature [11, 20]. Participants were provided with immediate access to permanent housing of their choice in their preferred neighbourhood (via rent supplements of $\$ 600 \mathrm{CAD}$ paid directly to the landlord), in addition to individualized and clientdriven mental health support services. Participants worked with a case manager to develop a participantdriven treatment plan, which included both immediate and long-term goals, such as application for disability benefits, access to primary care or other health services in the community, reconnecting with social networks, participation in substance misuse treatment programs and vocational training [21]. The staff ratio in the adapted HF team was 17:1 and services were provided for the duration of the follow-up.

The main principles of anti-racist/anti-oppressive service delivery have been outlined elsewhere $[11,20]$ and include: empowerment, education, alliance building, language use, alternative healing strategies, advocacy, social justice/activism and fostering reflexivity (critical self-knowledge, awareness and examination of one's social position and its influence) [20]. HF and anti-racist/ anti-oppressive practices share several elements, including a focus on client empowerment and choice, with HF additionally offering targeted strategies to ensure housing stability [11]. The agency offering the adapted 
HF intervention was committed to anti-racist/antioppressive frameworks of practice across program structures and offered regular staff training in such practices, as well as linguistic and culturally accessible programming and services, and a physical environment that is inclusive and welcoming to people from ethnic minority groups [11].

Anti-racist and anti-oppressive staff practices focus on breaking the silence about racism, addressing racism and discrimination, and examining power inequities, oppression and mental health together, taking anti-racist and anti-oppressive action as required [11,20]. Case managers embrace client-centred, strengths-based, holistic approaches to mental health, recognizing the importance of community of origin, family, and different ways of healing $[11,20]$. A key element of the adapted HF program is that case managers were representative of the population they served whenever possible and fluent in the primary language of program participants. In 2009, the agency offered services in 18 languages in addition to English. In addition to ICM services, the agency offered art and music therapy, a community kitchen, computer programs, life skills, traditional Chinese medicine, yoga, English as a second language, as well as support groups for men, women and youth $[11,22]$. Another key treatment approach adopted by the agency was the inclusion of families and peer networks early in the recovery process $[11,22]$.

Individuals randomized to the usual care group were able to access a variety of traditional housing programs, mental health and community services available in the city of Toronto and were provided with information on how to access such services. Toronto is a service rich environment with a variety of health and mental health services, as well as programs specifically serving people experiencing homelessness. Local services include crisis programs, drop-in centres, emergency shelters, inpatient/outpatient mental health services, meal programs, street outreach programs, supportive housing programs, comprehensive primary care teams, $\mathrm{ACT}$ and ICM teams [23]. In addition, a variety of specialized primary care and community mental health agencies exist which serve specific ethnic or language groups, immigrants and refugees [23].

\section{Data collection}

Participants were met every 3 months for face-to-face, structured, laptop computer-assisted interviews. Longer interviews took place at baseline, 6-, 12-, 18- and 24months. During each interview, data was collected and entered wirelessly directly into a secured central database. Brief call-in updates and interviews were conducted with participants on a regular basis to maintain contact and improve follow-up rates. Participants were financially compensated for all interviews and updates
(\$10 CAD for monthly calls, up to $\$ 40$ for short interviews and $\$ 60$ for longer interview). More details on study methodology and design for the At Home/Chez Soi study [10] and on this adapted program, can be found elsewhere [11].

\section{Outcomes}

The prespecified primary study outcome was housing stability, evaluated using the Residential Time Line Follow-Back (RTLFB) Inventory [24]. For each participant, we calculated the percent of days spent in stable housing during the 24-month follow-up period. Stable housing was defined as living either in one's own apartment, house or room or with family in addition to either tenancy rights or an expectation of residing at this location for $\geq 6$ months, as opposed to unstable housing, which included living on the street, temporary residences $(<6$ months' duration and/or no tenancy rights), shelters, crisis units and institutions.

Secondary outcomes explored mental and physical health, social outcomes and services use. Generic quality of life was assessed using the "overall health" Visual Analogue Scale (VAS) of the EuroQol 5 Dimensions (EQ-5D), which measures self-rated health status (both physical and mental) along a scale that ranges from worst (0) to best (100) imaginable health state [25]. Severity of problems with drugs or alcohol within the past month was assessed using the substance use screener (SDScr) of the GAIN Short Screener, GAIN-SS [26]. Additional questions asked about drug and alcohol use related problems and the amount of money spent on alcohol and drugs in the past-month. Psychiatric symptoms within the past month were evaluated using the 14-item modified Colorado Symptom Index (CSI), for which a total score was calculated [27]. Community functioning was evaluated via the total summary score of the MCAS [28]. The total score of the Quality of Life Index (QoLI-20), an instrument used widely in this population, was used for assessing condition-specific quality of life [29]. Physical and psychological community integration were assessed using the separate physical and psychological subscales of the Community Integration Scale (CIS) [30]. Emergency department use was assessed with two questions from the Health Service and Justice Service Use Questionnaire (HSJSU): i) "In the past 6 months, have you been to a hospital emergency room?" and ii) "Approximately how many emergency room visits did you have in total?" The number of days hospitalized was calculated from data collected from the RTLFB by examining the number of overnight stays in hospitals over the study period. The number of police arrests was captured by two additional questions from the HSJSU: i) "In the past six months, have you been arrested?", and ii) "How many times?". 


\section{Sample size calculation}

The primary outcome of housing stability was used to estimate a clinically meaningful effect size. In a previous randomized trial of supportive housing and ICM compared to standard care among homeless veterans with mental illness and/or substance use [31], participants spent an average of $66 \%$ and $53 \%$ of days housed (of past 90 days) in the intervention and usual care groups, respectively, with an estimated common group standard deviation as $26 \%$, resulting in a medium effect size (Cohen's $d=0.5$ ) [32]. In order to account for an assumed attrition rate of $40 \%$, we estimated that a sample size of 100 participants would be necessary in each arm in our study to provide $80 \%$ power to detect a medium effect size $(\mathrm{d}=0.5)$ [32] at 24 months using a two-sided $t$-test.

\section{Statistical analyses}

Missing data occurred in the key outcomes due to participant withdrawal, non-responses or refusal to answer, which we decided to impute a priori using sequential regression multivariate imputation (SRMI) [33]. This method allows for efficient imputation by fitting a model to each variable, conditional on all others, and imputing one variable at a time [34]. Variables included in the multiple imputation model included a) outcome variables collected at all study visits; b) study site; c) age at enrolment; d) gender; e) ethnic minority status and f) Aboriginal status. Imputed values were restricted to the theoretical range of the original variables by use of bounds. Using this approach, 40 imputed datasets were created using the mi impute chained command in STATA v.13 (StataCorp LP) and results were combined using PROC MIANALYZE in SAS 9.4 (SAS Institute). Because the extent of missing data was small (5\%), no imputation was performed for percent of days stably housed or the number of hospitalizations, which were calculated for the duration of the follow-up period for each participant for whom housing data was available, and who was known to be alive.

All statistical analyses were performed with SAS version 9.4 on an intention-to-treat basis. The primary outcome (percent of days stably housed during the 24month follow-up period) and the hospitalization outcome (rate of hospitalization during the 24 months follow-up period) were analysed by fitting mixed models, which assessed the main effect of treatment (adapted HF vs. usual care). For the percent of days stably housed, a linear mixed model was used (PROC MIXED), while a zero-inflated negative binomial (PROC GENMOD) model was fitted for the number of days hospitalized. For all other secondary outcomes for which longitudinal data was available, analyses were conducted using linear mixed models (PROC MIXED) for continuous outcomes and generalized linear models (PROC GENMOD) for count variables. The main effects of time and treatment as well as the interaction of treatment"time were examined. The unstructured covariance matrix for repeated measures was considered in all models. The significance level was set at $5 \%$ for all analyses.

In addition to the outcome analyses, we also examined what baseline variables were associated with the duration of being housed during the study period. In these analyses, the housing outcome was dichotomized into: a) the reference group, which was defined as those who spent less than half of the time $(<50 \%)$ in stable housing during the study period; and b) the group of interest, which was defined as those who had been stably housed half or more of the time ( $\geq 50 \%)$ during the 24-month study period. These secondary analyses employed a multivariate logistic regression analysis, and were conducted using a two-step process:

1. A list of baseline variables was established, based on potential associations with the outcome variables of interest, and included self-reported demographic variables (age, gender, total length of time homeless, education level, immigrant status, length of residency in Canada, native language), clinical variables (specific MINI diagnoses, including psychosis, major depressive disorder, post-traumatic stress disorder, alcohol or substance abuse or dependence) and the number of self-reported chronic health conditions [10]. Independent logistic models were performed for each baseline variable to test for associations. With the exception of the model examining the treatment variable, all other Step 1 models were adjusted for treatment group (i.e. these were bivariate tests). An $p<0.20$ was set for these preliminary Step 1 tests, as using an $p<0.05$ to examine potential confounders can lead to deletion of important confounder variables from the model [35].

2. All covariates that were identified as significant in Step 1 at $p<0.20$ were added into a multivariate logistic regression model. If two or more of the selected variables from Step 1 were highly correlated (e.g. age and length of time in Canada), we performed separate models with each of these variables, but only retrained one of the highly correlated variables in the multivariate model based on strength of effect in the model.

\section{Results}

Between October 28, 2009 and June 29, 2011, a total of 237 participants were recruited and randomized to either the adapted HF program $(n=135)$ or usual care $(n$ 
= 102) (Fig. 1). Follow-up interviews were completed for $90 \%(n=213)$ of participants at 12 months and $79 \%(n=$ 188 ) of participants at 24 months. By study end, 49 participants were lost to follow-up and one withdrew.

Baseline and demographic characteristics were similar between the groups (Table 1). Participants were mostly single and never married (69\%), male (67\%) and had been homeless for an average of 3.9 years SD 5.0 (median 2.1). While nearly half were native English speakers (48\%), more than two-thirds were born outside of Canada (72 \%). The most common ethnic groups reported were Black-Caribbean (22 \%), BlackAfrican (18 \%), Black-Canadian (14\%), Mixed (11\%) and South Asian (10\%). Based on the MINI, the most common diagnoses in our sample were depressive disorder (41\%), psychotic disorder (36\%), post-traumatic stress disorder $(24 \%)$ and mood-disorder with psychotic features $(23 \%)$. Substance use comorbidities were common, with more than one-third (34 \%) meeting criteria for alcohol abuse or dependence and $36 \%$ meeting criteria for substance abuse or dependence. Suicidality was common, with nearly one-third of our sample (29\%) reporting moderate to high suicidality.

During the 24-month study period, participants randomized to the adapted HF group were housed $75 \%$ (95\% CI 70-81) of the time, compared to usual care participants, who were housed $41 \%$ (95\% CI 35-48) of the time (difference $=34 \%, 95 \%$ CI 25 to 43). Results from a zero-inflated negative binomial model found that the number of self-reported days spent in hospital did not differ significantly between the treatment allocation groups (rate ratio $=1.9,95 \% \mathrm{CI} 0.78$ to 4.5 ).

Comparing 24 month values to baseline for secondary outcomes (Table 2), we only observed a significant treatment*time interaction for community functioning (MCAS), with greater improvements among participants

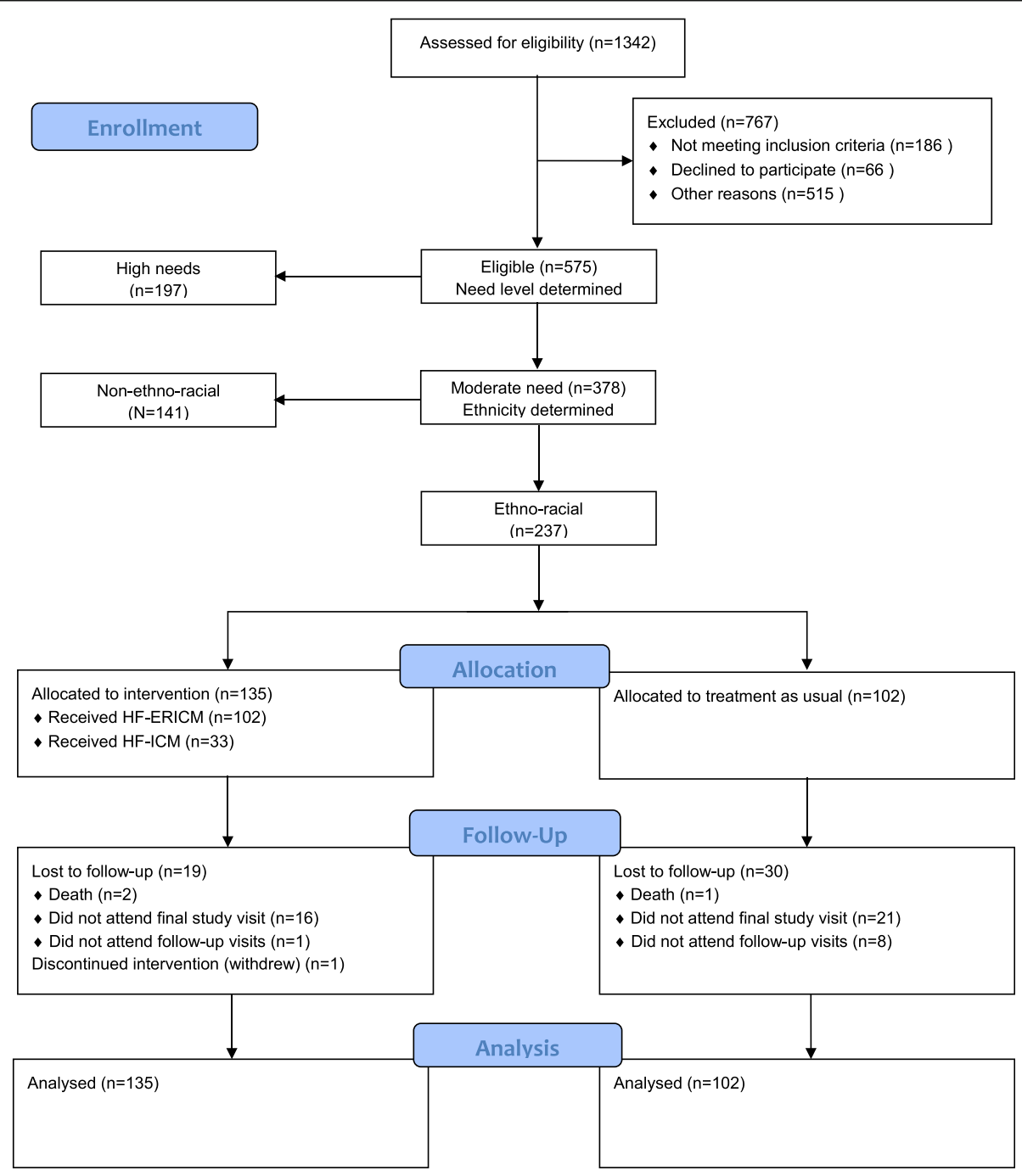

Fig. 1 Consort diagram showing participant flow through study 
Table 1 Participant demographics at study baseline ${ }^{a}$

\begin{tabular}{lll}
\hline Characteristic & Adapted & Usual care \\
& Housing First \\
$(n=135)$ & $(n=102)$ \\
& & \\
\hline
\end{tabular}

Age, $n(\%)$

$<30$

30-39

40-49

$\geq 50$

Male, $n(\%)^{b}$

Country of birth, $n(\%)$

Canada

$98(72.6)$

40 (29.6)

$28(27.5)$

$33(24.4)$

$21(20.6)$

$38(28.1)$

35 (34.3)

$24(17.8)$

18 (17.6)

$91(67.9)$

$65(66.3)$

Native language, $n(\%)$

English
Other

65 (48.1)

$70(51.9)$

$48(47.1)$

Ethnicity, n (\%)

Black - Caribbean Region

$32(23.7)$

$21(20.6)$

Black - Africa

Black - Canada

Mixed background ${ }^{c}$

South Asian

Other $^{d}$

Marital status $n$ (\%)

Single, never married

Other $^{\mathrm{e}}$

25 (18.5)

21 (15.6)

$9(6.7)$

$13(9.6)$

$35(25.9)$

$29(28.4)$

73 (71.6)

$54(52.9)$

$17(16.7)$

$13(12.7)$

$17(16.7)$

$10(9.8)$

$24(23.5)$

$89(66.4)$

$73(71.6)$

45 (33.6)

$29(28.4)$

Number of children, $n$ (\%)

0

1

$\geq 2$

$95(70.4)$

74 (72.5)

$24(17.8)$

$13(12.7)$

$16(11.8)$

15 (14.7)

Current housing status, $n$ (\%)

Absolutely homeless

$121(89.6) \quad 93(91.2)$

Precariously housed

Total length of homelessness, years, mean $\pm s d^{f}$

Longest period of homelessness, years, mean $\pm s d^{9}$

Education history, $n$ (\%)

$$
\begin{aligned}
& <\text { High school } \\
& \text { Completed high school }
\end{aligned}
$$

Some post-secondary school

MCAS total score, mean $\pm s d$

MINI results, $n(\%)^{\mathrm{h}}$

Depressive episode

Manic or hypomanic episode

Post-traumatic stress disorder

Panic disorder
$14(10.4) \quad 9(8.8)$

$3.5 \pm 4.4 \quad 4.4 \pm 5.7$

$1.9 \pm 3.0 \quad 2.2 \pm 2.8$

$22(16.3) \quad 23(22.5)$

$52(38.5) \quad 34(33.3)$

$65.5 \pm 3.12 \quad 65.4 \pm 3.25$

$57(42.2) \quad 39(38.2)$

$12(8.9) \quad 7(6.9)$

$34(25.2) \quad 23(22.5)$

$21(15.6) \quad 18(17.6)$
Table 1 Participant demographics at study baseline ${ }^{a}$

\begin{tabular}{|c|c|c|}
\hline Mood disorder with psychotic features & $30(22.2)$ & $24(23.5)$ \\
\hline Psychotic disorder & $46(34.1)$ & $40(39.2)$ \\
\hline Alcohol abuse or dependence & $43(31.9)$ & $38(37.3)$ \\
\hline Substance abuse or dependence & $45(33.3)$ & $41(40.2)$ \\
\hline Suicidality & $36(26.7)$ & $32(31.4)$ \\
\hline \multicolumn{3}{|c|}{$\begin{array}{l}\text { MCAS Multnomah Community Ability Scale, MINI Mini International } \\
\text { Neuropsychiatric Interview } \\
\text { aThe following variables had missing values: marital status }(n=1) \text {; total length } \\
\text { of homelessness ( } n=5 \text { ); longest period of homelessness }(n=1) \text {. Percentages } \\
\text { were calculated out of the total available data } \\
\text { bIndividuals who self-identified as female and other/transgendered/transsexual } \\
\text { are not listed due to small cell size } \leq 5 \text { for the latter category } \\
\text { c"Mixed" ethnicity included individuals who had at least one parent from the } \\
\text { following ethnicities: East Asian, South East Asian, African Black, Canadian } \\
\text { Black, Caribbean Black, Latin American, Indian-Caribbean and Middle Eastern } \\
\text { d'The "Other" category comprised of individuals who self-identified to one of } \\
\text { the following groups but were suppressed due to small cell size: East Asian, } \\
\text { Indian-Caribbean, Latin American, Middle Eastern and South East Asian } \\
\text { e"Other" marital status categories include the following options: married, } \\
\text { cohabitating with partner, divorced, separated, widowed } \\
\text { fMedian values in years for total length of homelessness were as follows: } \\
2.0 \text { for the adapted HF group and } 2.5 \text { for the usual care group } \\
\text { gMedian values in years for longest period of homelessness were as follows: } \\
1.0 \text { for the adapted HF group and } 1.0 \text { for the usual care group } \\
\text { hMINI Diagnoses all represent current diagnoses established at baseline } \\
\text { 'Suicidality was assessed as low, medium, or high; results here are shown for }\end{array}$} \\
\hline
\end{tabular}
(Continued)

in the adapted HF group, compared to those in the usual care group (change in mean difference $=2.2,95 \%$ CI 0.06 to 4.3). Although generic quality of life (EQ5D VAS) (change in mean difference $=6.9,95 \%$ CI 0.17 to 14.0), severity of substance use problems (GAIN) (ratio of rate ratios $=0.62,95 \% \mathrm{CI} 0.39$ to 0.98 ) and the number of days experiencing problems due to alcohol (ratio of rate ratios $=0.35,95 \%$ CI 0.14 to 0.88 ) improved significantly from baseline to 12 months among adapted HF compared to usual care participants; these treatment" time interactions were no longer statistically significant at 24 months. Additional file 1: Table S1 shows model-estimated outcome values at each study time point, by treat ment group.

The results of the unadjusted and adjusted logistic regressions for the secondary data analyses are found in Table 3. In total, $65 \%$ of participants were stably housed for $50 \%$ of the time or more. In preliminary bivariate logistic regression analyses (adjusting for treatment group) with $p<0.20$ (Table 3 , left column), male gender and MINI diagnosis of psychotic disorder were associated with reduced odds of being housed $50 \%$ of the time, while diagnosis of major depression and post-traumatic stress disorder were both associated with increased odds of being housed $50 \%$ of the time or more. These four variables were then included into the multivariate model, in addition to the treatment group.

In the final adjusted multivariate logistic model, with $p<0.05$ (Table 3, right column), participants allocated to the treatment group were almost eight times more likely 
Table 2 Treatment group changes from baseline ${ }^{a}$

\begin{tabular}{|c|c|c|c|c|}
\hline Outcome & 12 months & $P$ & 24 months & $P$ \\
\hline Health status (EQ5D-VAS) & $6.91(0.17$ to 13.66$)$ & 0.045 & $-0.12(-7.09$ to 6.84$)$ & 0.97 \\
\hline Mental illness symptomatology (CSI) & $-0.22(-3.42$ to 2.98$)$ & 0.89 & $0.04(-3.39$ to 3.46$)$ & 0.98 \\
\hline Substance use problem severity (GAIN-SS) & 0.62 (0.39 to 0.98$)$ & 0.04 & $1.00(0.61$ to 1.64$)$ & $>0.99$ \\
\hline Physical community integration (CIS-PHYS) & 0.98 (0.79 to 1.21$)$ & 0.84 & 0.86 (0.70 to 1.07$)$ & 0.17 \\
\hline Psychological community integration (CIS-PSYCH) & $0.27(-0.96$ to 1.49$)$ & 0.67 & $0.61(-0.64$ to 1.86$)$ & 0.34 \\
\hline Community functioning (MCAS) & $1.25(-0.72$ to 3.21$)$ & 0.21 & 2.16 (0.06 to 4.26$)$ & 0.04 \\
\hline Quality of life (QoLI) & $1.83(-4.70$ to 8.36$)$ & 0.58 & $2.94(-3.55$ to 9.42$)$ & 0.37 \\
\hline Number of emergency department visits & 0.74 (0.36 to 1.51$)$ & 0.41 & 0.67 (0.28 to 1.58$)$ & 0.36 \\
\hline Number of arrests & $1.66(0.51$ to 5.39$)$ & 0.40 & 1.31 (0.37 to 4.62$)$ & 0.67 \\
\hline Amount of money spent on alcohol in past 30 days & $-30.76(-80.58$ to 19.06$)$ & 0.23 & $-9.99(-47.62$ to 27.64$)$ & 0.60 \\
\hline Amount of money spent on drugs (not prescription) in past 30 days & $15.41(-132.47$ to 163.29$)$ & 0.84 & $84.32(-35.09$ to 203.74$)$ & 0.17 \\
\hline Number of days in past 30 experienced alcohol problems & 0.35 (0.14 to 0.88$)$ & 0.03 & $0.35(0.12$ to 1.02$)$ & 0.054 \\
\hline Number of days in the past 30 experienced drug problems & 0.73 (0.34 to 1.57$)$ & 0.43 & 0.58 (0.24 to 1.42$)$ & 0.23 \\
\hline
\end{tabular}

${ }^{a}$ The change from baseline to the other study time points corresponds to the mean change $(95 \% \mathrm{Cl})$ for continuous outcomes and the ratio of the rate ratios $(95 \% \mathrm{Cl})$ for count outcomes. $P$ values were assessed on the basis of the treatment group*time interaction. For continuous outcomes, the time by treatment group interaction examined the change in the mean from baseline to a subsequent follow-up visit (12- and 24-months) for the adapted HF group compared to the usual care group and $95 \% \mathrm{Cl}$. For count outcomes (substance use problem severity, physical community integration, number of emergency department visits, number of arrests, days experiencing problems due to alcohol, days experiencing problems due to drugs), the treatment group*time interaction evaluated the ratio of rate ratios for each post-baseline visit (e.g. rate ratio at follow-up visit relative to baseline in the adapted $\mathrm{HF}$ group divided by the rate ratio at follow-up visit relative to baseline in the usual care group) and $95 \% \mathrm{Cl}$. In these analyses, baseline values were used as a reference time point for all comparisons at subsequent time points (12- and 24-months) and the usual care group was used as a reference group

to be housed $50 \%$ or more of the time over the 24month study period (OR $=7.9,95 \%$ CI 4.1 to 15.2$)$ compared to those in the usual care group. Compared to those without a baseline diagnosis of psychosis, participants with this diagnosis had a reduced likelihood of being housed $50 \%$ or more of the time during the study period (OR $=0.37,95 \%$ CI 0.18 to 0.72$)$. Neither gender $(\mathrm{OR}=0.58,95 \% \mathrm{CI} 0.29$ to 1.6$)$, post-traumatic stress disorder $(\mathrm{OR}=1.3,95 \% \mathrm{CI} 0.58$ to 3.1$)$, nor major depression $(\mathrm{OR}=1.0,95 \% \mathrm{CI} 0.50$ to 2.1$)$ showed a statistically significant association with housing in adjusted analyses.

Table 3 Odds ratios from unadjusted and adjusted logistic regression models examining the effect of baseline measures on percent of time spent stably housed during 24 months of study ( $<50 \%$ of time vs. $\geq 50 \%$ of time)

\begin{tabular}{|c|c|c|c|c|}
\hline \multirow[t]{2}{*}{ Baseline variables } & \multicolumn{2}{|l|}{ Unadjusted $^{\mathrm{a}}$} & \multicolumn{2}{|l|}{ Adjusted } \\
\hline & OR (95 \% Cl) & $P$ & OR (95 \% Cl) & $P$ \\
\hline Treatment group & 7.17 (3.83 to 13.42$)$ & $<0.001$ & 7.86 (4.06 to 15.23$)$ & $<0.001$ \\
\hline Age (years) & 1.01 (0.98 to 1.04$)$ & 0.47 & & \\
\hline Gender & $0.57(0.29$ to 1.12$)$ & 0.10 & $0.58(0.29$ to 1.16$)$ & 0.12 \\
\hline Total length of homelessness (years) & $0.96(0.91$ to 1.02$)$ & 0.24 & - & - \\
\hline High School or higher education & $0.73(0.39$ to 1.37$)$ & 0.33 & - & - \\
\hline Immigrant Status & $0.92(0.46$ to 1.84$)$ & 0.82 & - & - \\
\hline Length of time in Canada & 0.99 (0.96 to 1.02$)$ & 0.36 & - & - \\
\hline English was first language & 1.07 (0.57 to 1.99$)$ & 0.83 & - & - \\
\hline \multicolumn{5}{|l|}{ MINI diagnoses } \\
\hline Psychosis & $0.34(0.17$ to 0.65$)$ & 0.001 & 0.37 (0.18 to 0.75$)$ & 0.01 \\
\hline Major depressive episode & 1.55 (0.81 to 2.96$)$ & 0.18 & $1.03(0.50$ to 2.10$)$ & 0.95 \\
\hline Alcohol or substance abuse or dependence & $1.10(0.59$ to 2.05$)$ & 0.77 & - & - \\
\hline Post-traumatic stress disorder & 1.75 (0.80 to 3.84$)$ & 0.16 & $1.32(0.58$ to 3.05$)$ & 0.51 \\
\hline Number of chronic medical conditions & $1.06(0.95$ to 1.18$)$ & 0.28 & - & - \\
\hline
\end{tabular}

${ }^{a}$ All univariate analyses were adjusted for treatment groups (adapted HF vs. usual care). Reference categories for categorical variables were as follows (indicated by 0 ): treatment group (usual care $=0$, adapted $\mathrm{HF}=1$ ); gender (female/other $=0$, male $=1$ ); education (less than high school $=0$; completed high school/some college/university = 1); immigrant status (Canadian born =0; foreign born =1); English as first language (other languages = 0 ; English = 1); MINI diagnoses (absence of diagnosis $=0$; presence of diagnosis $=1$ ) 


\section{Discussion}

Despite documented health disparities among people from black and ethnic minority groups, the literature on interventions aiming to improve health and social outcomes for this disadvantaged population is scant. People from black and ethnic minority groups experiencing homelessness and mental illness face additional challenges, as traditional housing and support strategies may not adequately address their needs. To our knowledge, this is the first study examining the effectiveness of a HF program which has been specifically adapted for this population. Enhancing HF with anti-racist/anti-oppressive principles of practice for black and ethnic minority homeless adults with mental illness successfully improved housing stability and community functioning for this population, compared to usual care, at the Toronto site of the At Home/Chez Soi trial. Our findings are consistent with previous studies of HF using rent supplements and mental health services (ACT or ICM) which report improvements in housing stability $[8,31$, 36], among homeless participants with mental illness. This study adds to this literature base by demonstrating the effectiveness of a HF adaptation, using anti-racist/ anti-oppressive practice, in improving housing stability among homeless adults with mental illness from ethnoracial minority groups, a population that may be reluctant to engage with traditional services and is at risk of poor outcomes $[1,2,5]$.

Interestingly, secondary analyses showed that diagnosis of psychosis was the only baseline variable examined found to be associated with housing stability over time. A previous single-site HF intervention that provided services via ICM found that psychotic symptom severity was similarly associated with leaving the project [37]. It is possible that the service intensity offered by ICMbased HF programs is not sufficient for some individuals experiencing psychotic symptoms, and that more intensive support services such as ACT may be needed for these participants.

We also observed that the adapted HF model led to improvements in community functioning among participants. Community functioning is a complex concept that encompasses symptom interference with functioning, adjustment to living, social competence and behavioural problems [17]. As part of the Community Mental Health Evaluation Initiative (CMHEI), participants with severe mental illness from three Canadian cities (Hamilton, Ottawa, Toronto) who were either homeless or at risk of homelessness were provided with various types of housing and supports $(n=91)$ and saw a significant improvement in MCAS scores from study baseline to 9-months of a magnitude of three points [38]. In the present study, treatment participants improved approximately four points from baseline, which was indicative of a small effect size (Cohen's $d=0.27$ ) [17, 39].

Unlike housing outcomes, substance use related outcomes have not shown consistent improvements among participants in HF programs. Reductions in alcohol use have been reported by a previous study examining a single-site HF intervention for adults with severe alcohol use disorders [40], but others, serving different subpopulations of homeless people, have found no changes in either drug or alcohol use after program enrolment [31]. The rates of alcohol or substance abuse or dependence (46\%) at study entry in our sample were comparable to a previous randomized trial of supported housing which also failed to see improvement in substance use problems (50\%) [31].

Previous reviews have confirmed that culturally adapted behavioural interventions result in better health outcomes compared to usual care or other controls [41]; however, few culturally adapted programs have been evaluated for superiority over the intervention they were adapted from, both because there is a lack of willingness to provide unaltered programs for cultural groups with known preferences (e.g. for language) but also due to the costs such comparisons would accrue [41]. The adapted HF program described here combines HF principles with anti-racist and anti-oppressive practice. The availability of this approach, in addition to more traditional HF programs, may offer ethno-racial minority participants a choice of mental health support services that may be more closely aligned with their personal preferences and support needs. Addressing experiences of racism and oppression may be an important factor in engaging in treatment and building a working alliance with some participants, which are important components of successful treatment outcomes [42]. Furthermore, increasing treatment choice may also lead to improved outcomes: a previous study of participants in a Housing First program reported that increased perceived choice is strongly inversely associated with psychiatric symptoms [43].

We have established the effectiveness of a HF program adapted for homeless adults with mental illness from black and ethnic minority groups compared to usual care. Future research should compare housing outcomes in traditional and adapted HF interventions to identify key ingredients of program success. As choice and plurality of services are important elements of healthcare in Canada, this study aimed to examine the effectiveness of the adapted HF intervention compared to usual services in the community. Our results therefore demonstrate the real world benefit of adapted HF in the Canadian context, supporting its inclusion in the complement of services and supports targeting black and ethnic minority groups. 
This study has some additional limitations. Firstly, the ethnic diversity in Toronto is more extensive compared to most large urban centres which therefore may not face similar challenges. Secondly, we have shown that the intervention works among this specific sample of individuals of ethnoracial ethnicity residing in Toronto, Canada. However, the unique ethno-cultural composition of our sample and the services available to the residents of Toronto may limit the generalizability of our findings to other populations of homeless adults with mental illness from different ethnic backgrounds and/or in other service delivery contexts. Third, this study uses self-reported data and therefore may be limited by recall bias and social desirability bias. Fourth, an economic evaluation has not yet been conducted, but will be the focus of future work. Fifth, while our targeted recruitment strategy attempted to capture a representative sample of homeless adults with mental illness from ethnic minority groups in Toronto, we cannot confirm that we succeeded in this goal due to the lack of accurate data on this difficult to reach population.

A key strength of this study is its ability to maintain high follow-up rates among a difficult to track population, which was greatly helped by monthly checks with participants and acquiring consent to contact service providers and family/friends for current participant contact information. More details on other strategies employed by study staff are described elsewhere [10,12].

Our findings add to the literature on cultural adaptations for groups experiencing severe disadvantage. Future studies comparing anti-racist/anti-oppressive adaptations to usual HF services and eliciting client preferences of and satisfaction with adapted services may better inform planning and resource allocation.

\section{Conclusions}

A Housing First intervention adapted using anti-racist and anti-oppression principles improved housing and community functioning outcomes in a diverse group of homeless ethnic minority participants with mental illness compared to usual care. The culturally adapted intervention may increase access to care through offering choice to racialized populations.

\section{Additional file}

Additional file 1: Table S1. Estimated outcome values at each study visit, by treatment group. (DOCX $13.9 \mathrm{~kb}$ )

\section{Abbreviations}

ACT: Assertive community treatment; AO: Anti-oppression; AR: Anti-racism; HF: Housing First; ICM: Intensive case management; MCAS: Multnomah community ability scale; MINI: Mini international neuropsychiatric interview

\section{Acknowledgements}

The authors would like to thank Rosane Nisenbaum and Kristen O'Brien for help with the multiple imputation and statistical analyses. We also thank Jayne Barker (2008-11), Ph.D., Cameron Keller (2011-12), and Catharine Hume (2012-present) Mental Health Commission of Canada At Home/Chez Soi National Project Leads, Paula Goering, Ph.D. The National Research Lead, the National Research Team, the five site research teams, the Site Co-ordinators, and the numerous service and housing providers, as well as persons with lived experience, who have contributed to this project and the research.

\section{Funding}

This research has been made possible through funding from the Mental Health Commission of Canada via financial contributions from Health Canada. The funder of the study had no role in study design, data collection, data analysis, data interpretation, or writing of this manuscript.

\section{Availability of data and materials}

The data supporting the conclusions of this article are not available in a public repository. However, a protocol is in place to provide access to the At Home/Chez Soi project data by external investigators once the appropriate ethics approvals and agreements are in place.

\section{Authors' contributors}

VS and KM designed the study. VS was the principal investigator and dratted the study protocol AG wrote the first draft of the report with input from VS. VM, AG and ASk performed statistical analyses. ASa and JC were managers of case management teams. AW was a study research assistant. VS takes responsibility of the integrity of the data and the accuracy of the data analysis. All authors contributed to, read and approved the final report.

\section{Competing interests}

The authors declare that they have no competing interests.

\section{Consent for publication}

Not applicable.

\section{Ethics approval and consent to participate}

The study was approved by the Research Ethics Board (\#09-208) of St. Michael's Hospital in Toronto, and was registered with the International Standard Randomized Control Trial Number (ISRCTN 42520374, assigned August 18, 2009). All study participants provided written informed consent.

\section{Author details}

${ }^{1}$ Centre for Addiction and Mental Health, Toronto, ON, Canada. ${ }^{2}$ Centre for Urban Health Solutions, St. Michael's Hospital, Toronto, ON, Canada. ${ }^{3}$ Department of Psychiatry, University of Toronto, Toronto, ON, Canada. ${ }^{4}$ Across Boundaries, Toronto, ON, Canada. ${ }^{5}$ Inner City Family Health Team, Toronto, ON, Canada. ${ }^{6}$ Health Equity Office, Centre for Addiction and Mental Health, room 2010, 33 Russell Street, Toronto, ON M5S 2G8, Canada.

Received: 31 May 2016 Accepted: 13 October 2016

Published online: 21 October 2016

\section{References}

1. U.S. Department of Health and Human Services (USDHHS). Mental Health: Culture, Race, and Ethnicity—A Supplement to Mental Health: A Report of the Surgeon General. Rockville: Department of Health and Human Services, Public Health Service, Office of the Surgeon General; 2001.

2. Hansson E, Tuck A, Lurie S, McKenzie K, for the Task Group of the Services Systems Advisory Committee Mental Health Commission of Canada. Improving mental health services for immigrant, refugee, ethno-cultural and racialized groups: Issues and options for service improvement. Calgary: Mental Health Commission of Canada; 2010.

3. National Institute of Mental Health-England (NIMHE). Inside outside: improving mental health services for Black and minority ethnic communities in England. Leeds: National Institute for Mental HealthEngland; 2003.

4. Horvitz-Lennon M, Zhou D, Normand SL, Alegria M, Thompson WK. Racial and ethnic service use disparities among homeless adults with severe mental illnesses receiving ACT. Psychiatr Serv. 2011;62(6):598-604. doi:10.1176/appi.ps.62.6.598. 
5. Horvitz-Lennon M, Frank RG, Thompson W, Baik SH, Alegria M, Rosenheck RA, et al. Investigation of racial and ethnic disparities in service utilization among homeless adults with severe mental illnesses. Psychiatr Serv. 2009; 60(8):1032-8. doi:10.1176/appi.ps.60.8.1032.

6. Fazel S, Geddes JR, Kushel M. The health of homeless people in highincome countries: descriptive epidemiology, health consequences, and clinical and policy recommendations. Lancet. 2014;384(9953):1529-40.

7. Aubry TP, Nelson GP, Tsemberis SP. Housing First for People With Severe Mental Illness Who Are Homeless: A Review of the Research and Findings From the At Home-Chez soi Demonstration Project/D'abord chez soi pour les personnes souffrant de maladie mentale grave qui sont sans abri : une revue de la recherche et des résultats du projet de démonstration At Home-Chez soi. Can J Psychiatr. 2015;60(11):467-74. doi:10.1136/bmjopen2011-000323. 10.1176/appi.ps.201200500.

8. Tsemberis S, Gulcur L, Nakae M. Housing First, consumer choice, and harm reduction for homeless individuals with a dual diagnosis. Am J Public Health. 2004;94(4):651-6.

9. Ridgway P, Zipple AM. The paradigm shift in residential services: From the linear continuum to supported housing approaches. Psychosoc Rehabil J. 1990;13(4):1. doi:10.1037/h0099479.

10. Goering P, Streiner D, Adair CE, Aubry T, Barker J, Distasio J, et al. The At Home/Chez Soi trial protocol: a pragmatic, multi-site, randomized controlled trial of Housing First in five Canadian cities. BMJ Open. 2011;1(2):e000323.

11. Stergiopoulos V, O'Campo P, Gozdzik A, Jeyaratnam J, Corneau S, Sarang A, et al. Moving from rhetoric to reality: adapting Housing First for homeless individuals with mental illness from ethno-racial groups. BMC Health Serv Res. 2012;12(1):345. doi:10.1186/1472-6963-12-345.

12. Hwang S, Stergiopoulos V, O'Campo P, Gozdzik A. Ending homelessness among people with mental illness: the At Home/Chez Soi randomized trial of a Housing First intervention in Toronto. BMC Public Health. 2012;12(1): 787. doi:10.1186/1471-2458-12-787.

13. BC Housing. Single Room Occupancy Hotels. Burnaby, British Columb. 2015. http://www.bchousing.org/Initiatives/Access/SRO. Accessed 2016 May 23.

14. American Psychiatric Association. The Diagnostic and Statistical Manual of Menta Disorders. 4th ed. Washington, DC: American Psychiatric Association; 1994.

15. Sheehan D, Janavs J, Harnett-Sheehan K, Sheehan M, Gray C, Lecrubier Y et al. M.I.N.I.: Mini International Neuropsychiatric Interview, English version 6.0.0 (DSM-IV). 2009.

16. Shelter Support and Housing Administration Division. 2006 Street needs assessment: Results and key findings. Toronto: City of Toronto; 2006

17. Barker S, Barron N, McFarland BH, Bigelow DA, Carnahan T. A community ability scale for chronically mentally ill consumers: Part II. Applications. Community Ment Health J. 1994;30(5):459-72.

18. Yau M, O'Reilly J. Research Report: 2006 Student Census, Grades 7-12 System Overview. Issued by the Organizational Development Department, Research and Information Services, Toronto Board of Education, Toronto, Ontario. 2007. http://www.tdsb.on.ca/Portals/0/aboutus/research/ 2006studentcensussystemoverview1.pdf. Accessed 2016 May 23.

19. Frane JW. A method of biased coin randomization, its implementation, and its validation. Ther Innov Regul Sci. 1998;32(2):423-32. doi:10.1177/ 009286159803200213

20. Corneau S, Stergiopoulos V. More than being against it: Anti-racism and anti-oppression in mental health. Transcult Psychiatry. 2012;49(2):261-82.

21. Polvere L, MacLeod T, Macnaughton E, Caplan R, Piat M, Nelson G et al. Canadian Housing First toolkit: The At Home/Chez Soi experience. Calgary and Toronto Mental Health Commission of Canada and the Homeless Hub; 2014.

22. Across Boundaries. Clarifying an Antiracism, Holistic Service Delivery Model in a Toronto Mental Health Agency. Toronto: Across Boundaries, An Ethnoracial Mental Health Centre; 2009.

23. City of Toronto. Guide to Services for People Who Are Homeless: Mental Health. 2016. http://www1.toronto.ca/wps/portal/contentonly?vgnextoid= d00ed4b4920c0410VgnVCM10000071d60f89RCRD\&vgnextchannel= cfa2d62869211410VgnVCM10000071d60f89RCRD. Accessed 2016 Sept. 7.

24. Tsemberis S, McHugo G, Williams V, Hanrahan P, Stefancic A. Measuring homelessness and residential stability: the residential time-line follow-back inventory. J Community Psychol. 2007;35(1):29-42.

25. Brooks R. EuroQol: the current state of play. Health Policy. 1996;37(1):53-72.

26. Dennis ML, Chan YF, Funk RR. Development and validation of the GAIN short screener (GSS) for internalizing, externalizing and substance use disorders and crime/violence problems among adolescents and adults. Am J Addict. 2006;15:s80-91.
27. Boothroyd $\mathrm{R}$, Chen $\mathrm{H}$. The psychometric properties of the Colorado symptom index. Adm Policy Ment Health. 2008;35(5):370-8.

28. Dickerson FB, Origoni AE, Pater A, Friedman BK, Kordonski WM. An expanded version of the Multnomah Community Ability Scale: anchors and interview probes for the assessment of adults with serious mental illness. Community Ment Health J. 2003;39(2):131-7.

29. Lehman AF. Measures of quality of life among persons with severe and persistent mental disorders. Soc Psychiatry Psychiatr Epidemiol. 1996;31(2): 78-88.

30. Segal SP, Aviram U. The mentally ill in community-based sheltered care: a study of community care and social integration. New York: John Wiley \& Sons; 1978

31. Rosenheck R, Kasprow W, Frisman L, Liu-Mares W. Cost-effectiveness of supported housing for homeless persons with mental illness. Arch Gen Psychiatry. 2003:60(9):940-51. doi:10.1001/archpsyc.60.9.940.

32. Cohen J. Statistical Power Analysis for the Behavioral Sciences. 2nd ed. NJ L. Erlbaum Associates: Hillsdale; 1988.

33. Graham J. Missing Data: Analysis and Design. Statistics for Social and Behavioral Sciences. New York: Springer; 2012

34. van Buuren S. Multiple imputation of discrete and continuous data by fully conditional specification. Stat Methods Med Res. 2007;16(3):219-42. doi:10.1177/0962280206074463.

35. Greenland S. Invited commentary: variable selection versus shrinkage in the control of multiple confounders. Am J Epidemiol. 2008;167(5):523-9. doi:10.1093/aje/kwm355.

36. de Vet R, van Luijtelaar MJA, Brilleslijper-Kater SN, Vanderplasschen W, Beijersbergen MD, Wolf JRLM. Effectiveness of case management for homeless persons: a systematic review. Am J Public Health. 2013;103(10): e13-26. doi:10.2105/ajph.2013.301491.

37. Collins SE, Malone DK, Clifasefi SL. Housing retention in single-site housing first for chronically homeless individuals with severe alcohol problems. Am J Public Health. 2013;103(S2):S269-S74. doi:10.2105/AJPH.2013.301312.

38. Nelson G, Sylvestre J, Aubry T, George L, Trainor J. Housing choice and control, housing quality, and control over professional support as contributors to the subjective quality of life and community adaptation of people with severe mental illness. Adm Policy Ment Health Ment Health Serv Res. 2007;34(2):89-100. doi:10.1007/s10488-006-0083-x.

39. Durbin J, Dewa CS, Aubry T, Krupa T, Rourke S, Foo E. The use of the multnomah community ability scale as a program evaluation tool. Can J Program Eval. 2004;19(3):135-57.

40. Larimer ME, Malone DK, Garner MD, Atkins DC, Burlingham B, Lonczak HS, et al. Health care and public service use and costs before and after provision of housing for chronically homeless persons with severe alcohol problems. JAMA. 2009;301(13):1349-57. doi:10.1001/jama.2009.414.

41. Barrera Jr M, Castro FG, Strycker LA, Toobert DJ. Cultural adaptations of behavioral health interventions: a progress report. J Consult Clin Psychol. 2013;81(2):196-205. doi:10.1037/a0027085

42. Baggett TP, O'Connell JJ, Singer DE, Rigotti NA. The unmet health care needs of homeless adults: a national study. Am J Public Health. 2010;100(7): 1326-33. doi:10.2105/AJPH.2009.180109.

43. Greenwood RM, Schaefer-McDaniel NJ, Winkel G, Tsemberis SJ. Decreasing psychiatric symptoms by increasing choice in services for adults with histories of homelessness. Am J Community Psychol. 2005;36(3-4):223-38. doi:10.1007/s10464-005-8617-z.

\section{Submit your next manuscript to BioMed Central and we will help you at every step:}

- We accept pre-submission inquiries

- Our selector tool helps you to find the most relevant journal

- We provide round the clock customer support

- Convenient online submission

- Thorough peer review

- Inclusion in PubMed and all major indexing services

- Maximum visibility for your research

Submit your manuscript at www.biomedcentral.com/submit 